\title{
Genetic analysis does not confirm non-classical congenital adrenal hyperplasia in more than a third of the women followed with this diagnosis
}

\author{
Valeria Alcantara-Aragon, ${ }^{1}$ Silvia Martinez-Couselo, ${ }^{2}$ Diana Tundidor-Rengel, ${ }^{1}$ \\ Susan M. Webb, ${ }^{1,5}$ Gemma Carreras, ${ }^{3}$ Juan J. Espinos, ${ }^{4}$ Ana Chico, ${ }^{1}$ \\ Francisco Blanco-Vaca, ${ }^{2}$ Rosa Corcoy ${ }^{1,6}$
}

${ }^{1}$ Endocrinology, ${ }^{2}$ Biochemistry, ${ }^{3}$ Pediatrics, and ${ }^{4}$ Gynecology Departments, Hospital de la Santa Creu I Sant Pau, ${ }^{5}$ CIBERER 747, ${ }^{6}$ CIBERBBN; Barcelona, Spain

Dear Sir,

Non-classical congenital adrenal hyperplasia (NC$\mathrm{CAH}$ ) due to 21-hydroxylase deficiency is one of the most frequent autosomal recessive diseases, with an estimated prevalence of 1 in 1000. It may manifest at different stages in life. In late childhood, it may present with advanced skeletal maturation, accelerated growth or premature pubarche. In adult women, the disease usually presents with a hyperandrogenic syndrome including hirsutism, acne, androgenic alopecia, anovulation, menstrual dysfunction, and infertility. ${ }^{1,2}$ However, not all individuals with NCCAH are symptomatic and affected males are not usually detected until a female family member is diagnosed., ${ }^{1,2}$

Individuals with NCCAH may be compound het-

Key words: 17 -alpha-hydroxy-progesterone, Non-classical congenital adrenal hyperplasia

Address for correspondence:

Valeria Alcantara-Aragon, Endocrinology Department, Hospital de la Santa Creu I Sant Pau, Sant Antoni Maria Claret 167, Barcelona 08025, Spain, Tel.: +30 935565661, Fax: +30 935565602, e-mail: VAlcantara@santpau.cat Received 03-03-2013, Accepted 14-04-2014 erozygotes and can carry severe $C Y P 21 A 2$ allele mutations. ${ }^{2}$ Consequently, genetic testing and counseling is highly recommended when planning pregnancy. Our aim was to assess CYP21A2 allele mutations and review the clinical characteristics in patients with a NCCAH diagnosis attended at the center, irrespective of their need for genetic counseling at short term.

Patients with a diagnosis of NCCAH were recruited when they came for their regular follow-up visits. Medical records were examined to retrieve data about initial clinical presentation, later signs and symptoms, hormonal work-up [basal and stimulated 17-hydroxyprogesterone (17OHP)], and treatment at diagnosis and follow-up. A standardized database was used. The interpretation of the patients' hormonal work-up was performed according to Speiser PW et al: basal $17 \mathrm{OHP} \geq 6$ and $<30 \mathrm{nmol} / \mathrm{L}$ was considered abnormal, while basal or stimulated $17 \mathrm{OHP} \geq 30$ and $<300 \mathrm{nmol} / \mathrm{l}$ was considered indicative of NCCAH. ${ }^{3}$ Analysis of the CYP21A2 gene was performed through polymerase chain reaction, sequencing, and family genetic testing when possible. ${ }^{4}$ Data obtained from descriptive statistic analysis is expressed as percentages and medians (interquartile range). Chi-square and Mann-Whitney tests were used for comparisons among groups. The Institutional Review Board gave special approval for the study. 
Results are summarized in Table 1. The audit included 29 women followed at the Pediatrics $(\mathrm{N}=5)$, Gynecology \& Obstetrics $(\mathrm{N}=6)$ or Endocrinology $\&$ Nutrition $(\mathrm{N}=18)$ Departments at our center. The initial clinical diagnosis was made in all of these departments, as well as in the Endocrinology Departments of other centers from 1976 to 2010. Twenty-four patients $(82.7 \%)$ were index cases. Age at first symptom was $15.8 \pm 8$ years and the median number of symptoms was 2 (1 to 3 ). The most common manifestations were hirsutism (69\%) and oligomenorrhea (48.3\%). Basal $17 \mathrm{OHP}$ at diagnosis was available in the clini-

Table 1. Summary of clinical information of women followed with a diagnosis of NCCAH

\begin{tabular}{lcc}
\hline Patient characteristics: & N or mean & \% or IQR \\
\hline Index case & 23 & 79.3 \\
Age at first symptom (years, mean + SD) & 15.8 & 8 \\
Number of symptoms, median (IQR) & 2 & 1 to 3 \\
\hline Most common manifestations: & $\mathbf{N}$ & $\mathbf{\%}$ \\
\hline Hirsutism & $\mathbf{2 0}$ & 69 \\
Oligomenorrhea & 14 & 48.3 \\
Infertility & $\mathbf{4}$ & 13.8 \\
Accelerated linear growth & $\mathbf{4}$ & 13.8 \\
Acne & $\mathbf{3}$ & 10.3 \\
Alopecia & $\mathbf{3}$ & 10.3 \\
Precocious puberty & $\mathbf{3}$ & 10.3 \\
\hline
\end{tabular}

\begin{tabular}{|c|c|c|}
\hline Hormonal work-up at diagnosis: & Availability, n (\%) & Concentration (nmol/l), median (IQR) \\
\hline Basal 17 OH-P & $22(75.9)$ & $14(5.75$ to 41$)$ \\
\hline ACTH stimulated 17 OH-P & $8(27.6)$ & $142(38.5$ to 177.75$)$ \\
\hline Pharmacological treatment \# & $\mathbf{N}$ & $\%$ \\
\hline None & 38 & 12.1 \\
\hline Corticosteroids: & 233 & 74.2 \\
\hline Dexamethasone & 188 & 59.8 \\
\hline Hydrocortisone & 44 & 14 \\
\hline Prednisone & 1 & 0.3 \\
\hline Other treatments, alone or a combination: & 43 & 13.7 \\
\hline Oral contraceptives & 27 & 8.5 \\
\hline Spironolactone & 15 & 4.8 \\
\hline Flutamide & 1 & 0.31 \\
\hline Genetic analysis: & $\mathbf{N}$ & $\%$ \\
\hline No mutations & 3 & 10.3 \\
\hline Heterozygous, mild mutation & 8 & 27.6 \\
\hline Homozygous, mild mutation & 9 & 31 \\
\hline Compound heterozygous, mild/severe & 9 & 31 \\
\hline Most frequent mutations: & $\mathbf{N} \ddagger$ & $\%$ \\
\hline Mild exon 7, Val281Leu & 28 & 62 \\
\hline Mild exon 10, Pro453Ser & 6 & 11.1 \\
\hline Deletion & 5 & 8.8 \\
\hline Severe exon 10, R483P & 3 & 6.6 \\
\hline Severe IVS2, 290-13 C> G & 2 & 4.4 \\
\hline Severe exon 7, His282Tyr & 1 & 2.2 \\
\hline
\end{tabular}

IQR: Interquartile range; \# in 314 follow-up visits; $\ddagger$ One patient had 3 mutations. 
cal records of 22 patients (75.9\%) and was $14 \mathrm{nmol} / \mathrm{l}$ (5.75 to 41). Stimulated 17OHP was available in 8 patients (27.6\%) and was $142 \mathrm{nmol} / \mathrm{l}$ (38.5 to 177.75$)$. In a substantial subset of patients in follow-up for NCCAH $(16,55.2 \%)$, hormonal work-up available in the clinical records was not sufficient to establish a NCCAH diagnosis.

Genetic analysis is summarized in Table 1. Genetic testing of parents or siblings was performed in 10 patients $(34.5 \%)$ to clarify the genetic diagnosis. The most common mutation ( 28 out of $45,62 \%$ ) was one that is known to be mild: Val281Leu. Globally, $37.9 \%$ of the genetic results did not confirm the alleged diagnosis. Other genes were not analyzed, but since $95 \%$ of cases are due to a mutation of the CYP21A2 gene, ${ }^{3}$ it is unlikely that more than one third of the women had a mutation in a different gene.

The rate of non-confirmatory genetic analysis was not significantly different in women with a satisfactory biochemical diagnosis of NCCAH (33.1\%) vs those without (50\%). Department of origin, the patient's index condition, age at initial presentation, and number of signs/symptoms did not differ between women with and without a genetic diagnosis of NCCAH (data not shown). Twenty-six women $(89.7 \%)$ received drug treatment for NCCAH at some point during follow-up, most frequently corticosteroids (23 women, $79.3 \%$ ). Similarly, at 314 follow-up visits, $87.9 \%$ of the women were receiving drug treatment, most frequently corticosteroids (74.2\%). Use of drug treatment did not differ in women with and without a genetic diagnosis of NCCAH ( 88.9 vs $90.9 \%$ at some point during follow-up, ns).

The main finding of this investigation is that a clinically significant subset of women followed and treated according to a diagnosis of NCCAH did not have a confirmatory genetic diagnosis. Some patients had been referred from other centers and access to lab tests at diagnosis and follow-up was limited. This could partly explain the less than optimal rate of available hormonal work-up at diagnosis. The limited sample size may have contributed to the non-significant differences in the characteristics of women with and without a genetic diagnosis of NCCAH.
All women included in this series presented symptoms suggestive of NCCAH together with at least an abnormal basal 17OHP at some point that could help understand the assignment of a NCCAH diagnosis. The heterozygote condition was not uncommon. In the literature, the clinical presentation and $17 \mathrm{OHP}$ values of the heterozygote carriers may overlap with that of NCCAH. ${ }^{2}$ Other conditions, such as premature adrenarche in childhood or PCOS in young adults, might have been present. For example, Pall et $\mathrm{al}^{5}$ reported an elevation of basal $17 \mathrm{OHP}$ in up to $25 \%$ of patients with PCOS.

Current guidelines emphasize limiting drug treatment to symptomatic NCCAH patients and avoiding corticosteroids whenever possible, with the exception of women seeking pregnancy. ${ }^{3}$ At present we can only speculate on the potential untoward effects of prolonged treatment for a non-existing NCCAH.

The results of this audit compel us to reconsider the diagnostic and therapeutic requirements of patients coming to the outpatient clinic with an alleged diagnosis of NCCAH. The fact that a significant percentage of patients attended in different departments with a NCCAH diagnosis did not have a confirmatory genetic analysis suggests that the situation may not be uncommon.

\section{REFERENCES}

1. White PC, Speiser PW, 2000 Congenital adrenal hyperplasia due to 21-hydroxylased Deficiency. Endocr Rev 21: 245-291.

2. Witchel SF, 2013 Non-classic congenital adrenal hyperplasia. Steroids 78: 747-750.

3. Speiser PW, Azziz R, Baskin LS, et al, 2010 Congenital Adrenal Hyperplasia Due to Steroid 21-Hyroxylase Deficiency: An Endocrine Society Clinical Practice Guideline. J Clin Endocrinol Metab 95: 4133-4160.

4. Bidet M, Bellanné-Chantelot C, Galand-Portier MB, et al, 2009 Clinical and molecular characterization of a cohort of 161 unrelated women with nonclassical congenital adrenal hyperplasia due to 21-hydroxylase deficiency and 330 family members. J Clin Endocrinol Metab 94: 1570-1578.

5. Pall M, Azziz R, Beires J, et al, 2010 The phenotype of hirsute women: a comparison of polycystic ovary syndrome and 21-hydroxylase-deficient nonclassic adrenal hyperplasia. Fertil Steril 94: 684-689. 